\title{
Delineating the electrophysiological signature of dystonia.
}

\author{
Anna Latorre ${ }^{1}$, Lorenzo Rocchi ${ }^{1}$, Kailash P. Bhatia ${ }^{1}$ \\ ${ }^{1}$ Department of Clinical and Movement Neurosciences, UCL Queen Square Institute of Neurology, \\ University College London, WC1N 3BG, London, United Kingdom
}

\section{Corresponding author:}

Professor Kailash P. Bhatia

Department of Clinical and Movement Neurosciences

UCL Queen Square Institute of Neurology

Queen Square, London WC1N 3BG, United Kingdom

E-mail:k.bhatia@ucl.ac.uk

Word count: 25869 characters with spaces

Running title: The electrophysiological signature of dystonia

Key words: dystonia, inhibition, plasticity, cerebellum, electrophysiology

\begin{abstract}
Over the last 30 years, the concept of dystonia has dramatically changed, from being considered a motor neurosis, to a pure basal ganglia disorder, to finally reach the definition of a network disorder involving the basal ganglia, cerebellum, thalamus, and sensorimotor cortex. This progress has been possible thanks to the collaboration between clinicians and scientists, and the development of increasingly sophisticated electrophysiological techniques able to non-invasively investigate pathophysiological mechanisms in humans. This review is a chronological excursus of the electrophysiological studies that laid the foundation for the understanding of the pathophysiology of dystonia and delineated its electrophysiological signatures. Evidence for neurophysiological abnormalities are grouped according to the neural system involved, and a unifying theory, bringing together all the hypothesis and evidence provided to date, is proposed at the end.
\end{abstract}




\section{Introduction}

At the beginning of the $20^{\text {th }}$ century, under the influence of the recent development of the psychoanalytic theory, neurology and psychiatry evolved into separate disciplines: the former focussed on brain diseases with a recognizable organic component, while the latter on pathologic conditions thought to originate from the mind. The subtle boundary between the two favoured the development of conflicting theories about aetiology and pathogenesis of certain disorders. This is true for dystonia which, at the time of the schism, was retained amongst organically unaligned or functional conditions and then classified as a motor neurosis (Newby et al. 2017). David Marsden, with his brilliant intuition, removed dystonia from this unclear classification and recognized it as a basal ganglia (BG) disorder. Marsden's concept was based on two main arguments: 1) the clinical observation of typical dystonic pattern in the context of damage to the BG and 2) the evidence of physiological abnormalities by using non-conventional electrophysiologic techniques, which pointed towards the loss of normal BG inputs onto spinal and brainstem interneurons (Marsden and Rothwell 1987). It was, in fact, the implementation of new neurophysiological investigations and the encounter between a great clinician and scientist, David Marsden, and an enthusiastic physiologist, John Rothwell, that laid the foundation for the understanding of the pathophysiology of dystonia.

In this review we chronologically retraced the steps that, over 30 years, have led to the understanding of the pathophysiological mechanisms causing dystonia, investigated with electrophysiological techniques. To facilitate reading, evidence for neurophysiological abnormalities are grouped according to the neural system involved. We conclude discussing the most recent concepts on the pathophysiology of dystonia and by proposing a unifying theory that brings together all the hypotheses and evidence provided to date.

\section{Spinal cord and brainstem}

The classical electromyographic (EMG) pattern of dystonia often consists of a simultaneous activation of agonist and antagonists muscles, described as co-contraction (Rothwell et al. 1983); therefore, abnormalities in the spinal cord circuitry were first considered as possible pathophysiological hallmarks of dystonia. Stretch reflex, $\mathrm{H}$-reflex, reciprocal inhibition of $\mathrm{H}$-reflex and ballistic movements at a single joint were therefore investigated. In summary, the results of these early studies showed increase in duration of both long-latency stretch reflex and the first burst of agonist EMG activity in ballistic arm movements (Rothwell et al. 1983; Marsden and Rothwell 1987), reduction in the amount of presynaptic inhibition in the spinal cord (Rothwell et al. 1988; Nakashima et al. 1989), and overflow 
of activity to muscle not directly involved in the action during voluntary and reflex movements (Rothwell et al. 1983). These results suggest a general lack of inhibition in both reflex and voluntary movements in dystonia, likely due to defective descending control on spinal cord (Marsden and Rothwell 1987; Sheehy et al. 1988). Similar conclusions have been drawn studying brainstem reflexes in patients with different forms of dystonia. Although the reflex arcs and the neural structures which mediate the blink and corneal reflexes were considered to be intact, one study showed an abnormal excitatory drive to the facial motoneurons and interneurons mediating facial reflexes in patients with blepharospasm and oromandibular dystonia (Berardelli et al. 1985). Also, the recovery cycle of the blink reflex, which assesses brainstem excitability, was found to be abnormal, due to reduced inhibition of the R2 component, mainly in patients affected by blepharospasm (Berardelli et al. 1985; Schwingenschuh et al. 2011), but also in segmental/generalized dystonia or torticollis, and not in those with focal arm dystonia; this suggested that the extent of dystonia and its proximity to the cranial muscles might be important in determining abnormal interneuronal function (Nakashima et al. 1990).

\section{Basal ganglia}

Although interneuronal abnormalities were observed at the spinal cord and brainstem level, the primary cause of dystonia was still thought to reside in the BG (Marsden and Rothwell 1987). This hypothesis came from the observation that secondary dystonia is most often due to lesions of the BG or thalamus and that it is frequently part of syndromes which entail BG pathology (Marsden and Rothwell 1987). According to the first model proposed to explain the involvement of BG in dystonia, the direct pathway is hyperfunctional, while the indirect one is hypofunctional, as a consequence of reduced activity along putamen-external globus pallidus (GPe) connections and increased inhibition of the subthalamic nucleus (STN) and internal globus pallidus (GPi) by the GPe; this abnormalities would result in reduced inhibition of the thalamus and increased excitation of the cortex (DeLong 1990; Mitchell et al. 1990). Invasive human studies have shed some light on the neuronal activity of BG in dystonia. Single-cell recording in patients undergoing functional neurosurgery demonstrated several abnormalities in the activity of GPe and GPi neurons, consisting in a decreased discharge rate (Vitek 2002; Starr et al. 2005) and overall irregular firing (Vitek et al. 1999; Hutchison et al. 2003; Starr et al. 2005; Vitek et al. 2011). These changes contribute to dystonia, as confirmed by the fact that deep brain stimulation (DBS) improves dystonia by restoring more physiological firing patterns in the GPi (Barow et al. 2014). Another electrophysiological abnormality in the BG of dystonic patients is represented by an excess of synchronized low-frequency $(4-10 \mathrm{~Hz})$ activity (Silberstein et al. 2003). The finding of high coherence between this abnormal oscillations and involuntary EMG activity in dystonic muscles suggested that excessive low-frequency oscillatory activity in BG is causally involved in 
dystonia (Chen et al. 2006; Neumann et al. 2015). Abnormal bursting and oscillatory activity might not be specific for dystonia, as similarities have been observed with Parkinson's disease (PD); however, these would be superimposed on a low background discharge rate in dystonia, whereas the latter would be higher in PD (Starr et al. 2005). It is to note that a decrease in BG output in dystonia has not always been confirmed (Hutchison et al. 2003), likely due to different dystonia phenotypes studied; it has been proposed that the final common pathway linking the different forms of dystonia needs to be found in cortical processing (Wichmann and Dostrovsky 2011).

\section{Motor cortex}

The BG are part of a group of parallel closed circuits that originate in the cerebral cortex, traverse the thalamus, and then project back to the cortical areas of origin (DeLong 1990). Cortical motor areas are one of the primary projection target of the BG (i.e. the motor loop) and the concept of a possible involvement of the inhibitory circuits within the primary motor cortex in dystonia was thought to be reasonable. Moreover, the introduction of transcranial magnetic stimulation (TMS) encouraged researchers to non-invasively explore cortical excitability in humans.

In the pioneering study of Ridding et al. (1995), paired-pulse TMS, consisting of a subthreshold conditioning stimulus preceding a suprathreshold test stimulus, at an interstimulus interval (ISI) of 1$6 \mathrm{~ms}$, was applied over the primary motor cortex (M1) of patients with focal task specific dystonia, to investigate M1 inhibitory intracortical circuits (Ridding et al. 1995b). Dystonic patients showed significant less inhibition compared to healthy controls. This paradigm is now known to be mediated by GABAa receptors and is called short-interval intracortical inhibition (SICI) (Chen et al. 2008). Later studies strengthened this result and showed that reduced intracortical inhibition not only contributes to dystonia, but also predispose its development. For instance, both manifesting and non-manifesting gene carriers of the most common form of generalised dystonia (DYT1 gene mutation) had reduced $\mathrm{SICl}$ and shortened cortical silent period (SP)(Edwards et al. 2003), which is another TMS measure of intracortical inhibition likely mediated by GABAb receptors (Chen et al. 2008). SICI was found to be reduced also in patients with functional dystonia; this abnormality was considered to be secondary to the prolonged abnormal posture of the affected body part (Espay et al. 2006). However, this would not explain the presence of reduced SICl in non-manifesting DYT1 carries (Edwards et al. 2003) and in unaffected body parts of suspected functional patients with fixed dystonia (Avanzino et al. 2008). Therefore, the role of $\mathrm{SICl}$ in dystonia is still not completely clear, but it is possible that it predisposes to the development of a dystonic phenotype and that additional factors (for instance genetic or psychological) are needed to develop the symptoms (Edwards et al. 2003; Latorre et al. 2019). Longinterval intracortical inhibition, a measure believed to involve GABAb receptor neurotransmission, can 
be normal in dystonia at rest (Meunier et al. 2012), but reduced during movements (Chen et al. 1997). Another measure of inhibition, namely interhemispheric inhibition $(\mathrm{IHI})$, was found to be reduced in dystonia, especially in those with mirror phenomenon (Beck et al. 2009; Nelson et al. 2010; Sattler et al. 2014). IHI refers to the inhibitory control of one brain hemisphere over the other, mediated by transcallosal fibres, and represent a marker of bimanual control; in dystonia the abnormal IHI may contribute to the overflow of muscle activities and mirror dystonia.

One of the distinguished features of dystonia is that it can affect highly skilled individuals (such as musicians) as a possible result of maladaptive reorganisation of neuronal structures caused by overtraining. It is therefore possible that plastic cortical changes may reinforce an intrinsic deficit and trigger symptoms onset in predisposed subjects (Rothwell and Huang 2003). In fact, dystonia caused by excess practice does not occur in everyone, but it has been proposed to occur only in individuals with possible pre-existing abnormalities in cortical function (Quartarone et al. 2006). This is supported, for instance, by the evidence that patients with focal hand dystonia, despite unilateral symptoms, show bilateral abnormalities in the somatosensory and motor systems (as demonstrated in behavioural (Fiorio et al. 2003), magnetoencephalography (Meunier et al. 2001), MRI (Garraux et al. 2004) and TMS (Ridding et al. 1995a) studies); since these dysfunctions occur in body areas not affected by dystonia, they are likely to reflect a primary brain dysfunction. Sensorimotor cortical plasticity was found abnormally increased in writer's cramp using paired associative stimulation (PAS), a TMS technique able to induce long-term potentiation (LTP)-like phenomena (Quartarone et al. 2003). The cause of this dysfunction was assumed to be related to the mechanisms that counteract the destabilizing influence of synaptic plasticity and, hence, stabilize neural activity within a physiologically meaningful range, i.e. homeostatic plasticity. To confirm this hypothesis a method to probe homeostatic plasticity was used in writer's cramp patients (Quartarone et al. 2005). It consisted of applying transcranial direct current stimulation (tDCS), which can facilitate or inhibit motor excitability according to its direction (i.e. anodal and cathodal), to precondition the response of the motor cortex to a subsequent period of inhibitory low-frequency repetitive transcranial magnetic stimulation (rTMS). Differently from healthy subjects, rTMS induced no consistent changes in corticospinal excitability after preconditioning by either form of tDCS in patients, indicating a loss of the normal homeostatic response pattern.

Abnormal synaptic plasticity was therefore proposed to be the key mechanism that underlies the development of the symptoms. As a matter of fact, while other electrophysiological measures (like intracortical inhibition and sensory abnormalities) are similarly abnormal in manifesting and nonmanifesting individuals with inherited primary dystonia (DYT1 gene mutation), susceptibility to plastic change in response to TMS stimuli appears to differentiate such individuals (Edwards et al. 2006). Also, 
abnormal plasticity distinguishes organic from functional dystonia (Quartarone et al. 2009). Accordingly, although reduced inhibition and possibly sensory abnormalities predispose to the development of dystonia, maladaptive plasticity seems to be more closely linked to the clinical emergence (Edwards et al. 2006).

A direct consequence of these observations is that synaptic plasticity modulation could be potentially used to treat dystonia. For instance, it has been shown that reducing cortical excitability in patients with dystonia using rTMS can produce corresponding changes in abnormal spinal motor output (Huang et al. 2004). Interestingly, DBS to the GPi, an effective treatment for primary dystonia, has its maximum clinical effect weeks or months after surgery, differently from PD, in which it takes only hours. This slowly progressive change might be due to a process of neural reorganization. In fact, while after DBS, SICl, reciprocal inhibition and blink reflex inhibition progressively normalize, in parallel to the clinical improvement, synaptic plasticity has a different course: it is virtually abolished at one month and then increases towards normal values over the following months (Ruge et al. 2011b). This implies that, when DBS is turned on, it rapidly removes abnormal BG signals (resulting in absent response to plasticity-inducing paradigms), but it takes time to cancel the persisting "memory" of abnormal movement pattern and to restore more normal patterns of activity. This observation suggests that plasticity determines the long-term therapeutic effects of DBS in dystonia. Accordingly, it was demonstrated that DBS induces long-term neural reorganization in the motor system, with a significant variation among patients depending on the plastic response (Ruge et al. 2011a). A good plastic response reflects a longer-term retention of normal movement patterns after treatment.

\section{Somatosensory cortex}

Since dystonia was recognized as a motor disease, clinicians and researchers have always been fascinated by a peculiar phenomenon: the relief of dystonic activity by touching the body segment affected by it. This phenomenon is known as "sensory trick" or "geste antagoniste". Such observation suggests that dystonia is influenced by tactile and proprioceptive sensory inputs and, if so, that abnormal muscle activity might be controlled by modulating afferent information. In one of the early studies on writer's cramp it was shown that increasing and decreasing muscle spindle activity by means of vibration or intramuscular injection of lidocaine, resulted respectively in dystonic movements of the limbs and dystonia suppression (Kaji et al. 1995). Despite the results confirmed that muscle afferents may contribute in producing dystonic posture, to understand the role of proprioceptive input additional data is required. Conversely, more attention has been given to the mechanisms that underlie sensory information processing and sensorimotor integration in dystonia. For instance, it was found that patients with writer's cramp lack the normal gating of afferent 
information due to a disordered central control of afferent inputs (Murase et al. 2000). Moreover, an abnormal somatotopic digital representation in the primary somatosensory cortex (S1) has been demonstrated in focal hand dystonia(Bara-Jimenez et al. 1998; Elbert et al. 1998; Bara-Jimenez et al. 2000; Meunier et al. 2001), but it is still not clear whether this finding represents a primary pathological process of dystonia or is a consequence of other phenomena, such as abnormal sensory inputs/dedifferentiation of sensory feedback from the affected body part, or repetitive dystonic movements .

The principal evidence of the sensory involvement in dystonia is that of abnormal tactile information processing in both the spatial and temporal domains (Tinazzi et al. 2006; Conte et al. 2019). Indeed, at a behavioural level, the most consistent sensory abnormality of dystonia is increased somatosensory temporal discrimination threshold (STDT), a measure of the acuity of temporal processing of sensory information defined as the shortest interval at which two tactile stimuli delivered to the same body part can be recognized as temporally separated (Conte et al. 2019). Increased STDT in dystonia is related to reduced activity of inhibitory circuits within S1 (as demonstrated by the correlation between high STDT and reduced suppression of somatosensory evoked potentials recovery cycle and reduced area of high-frequency oscillations), and assumed to be the result of specific abnormalities within circuits processing the temporal aspects of afferent inputs (Antelmi et al. 2017). STDT is not ameliorated by GPi DBS (Sadnicka et al. 2013), supporting the hypothesis that sensory abnormalities in dystonia might reflect the functional derangement of a network involving BG-thalamus-sensorimotor cortex.

The idea of reversing dystonia's pathophysiological abnormalities by inducing plastic changes was also applied to the somatosensory cortex. With the intent of enhancing the defective inhibitory mechanisms in the sensorimotor cortex, and therefore normalise the processing of the somatosensory input and possibly improve symptoms, a peripheral non-invasive stimulation, known as highfrequency repetitive somatosensory stimulation (HF-RSS), was applied in dystonic patients (Erro et al. 2018). HF-RSS is a patterned electric stimulation applied to the skin through surface electrodes, which has been demonstrated to enhance intracortical inhibitory mechanisms within S1 and M1 (Erro et al. 2016; Rocchi et al. 2017). These effects are a considered a form of short-lasting synaptic plasticity. Patients with cervical dystonia, however, showed a paradoxical response to HF-RSS, as it reduced, instead of increasing, inhibition within the sensorimotor cortex. According to the authors, this reverse plastic response was likely due to abnormal homeostatic inhibitory plasticity within the sensorimotor cortex (Erro et al. 2018).

\section{Cerebellum}


Reports of cerebellar lesions causing dystonia have raised the question of a cerebellar involvement in its pathophysiology and encouraged researcher to investigate it further. The two principal cerebellar functions studied in dystonia are associative motor learning and motor adaptation.

Eyeblink classic conditioning (EBCC) is an associative learning protocol in which a sound is conditioned to provoke an eyeblink in the absence of the unconditioned stimulus, that is an electric shock to the supraorbital nerve. The EBCC is related to the integrity of the olivo-cerebellar circuit and relies on integration of afferent information at the level of Purkinje cells and deep cerebellar nuclei (Monaco et al. 2018). EBCC is impaired in patients with primary focal dystonia (Teo et al. 2009; Kojovic et al. 2013), but it is normal in patients with generalised and segmental inherited dystonia caused respectively by DYT1 and DYT6 gene mutation (Sadnicka et al. 2015). This discrepancy might suggest that the cerebellar contributions differs according to the form of dystonia. However, Antelmi and coworkers pointed out that these conflicting results might be due to the fact that focal forms of dystonia are often associated with tremor, which could have been overlooked in early studies (Antelmi et al. 2016). The authors demonstrated, in fact, that EBCC segregate with tremulous dystonia, whereas dystonic patients without tremor show normal conditioning. In conclusion, it is possible that tremor in dystonia may be linked to dysfunction in cerebellar circuits mediating EBCC.

The ability to overcome movement perturbations caused by novel environment or altered sensory feedback, when performing a motor task, is called motor adaption and depends on cerebellar function. Motor adaptation is not impaired in patients with cervical dystonia, when assessed by visual and proprioceptive sensorimotor integration tasks, regardless the presence of tremor (Sadnicka et al. 2014b). Associative learning and motor adaptation are distinct functions of the cerebellum, expressive two different forms of motor learning. This might explain the different results obtained, however, to clarify their involvement in dystonia pathophysiology more data are needed.

Finally, minor evidence on functional impairment of cerebellar modulation on the motor cortex in dystonia comes from the observation of absent cerebellar-brain-inhibition, measured by TMS, in dystonic patients (Brighina et al. 2009).

\section{Conclusion: the network model}

Thanks to the combination of careful clinical observation and detailed electrophysiological investigations, in about 3 decades, our concept of dystonia has radically changed, and its pathophysiological features have been delineated (Table 1). Initially, dystonia was thought to be a psychiatric disorder, but evidence of reduced inhibition at different levels of the central nervous system, increased sensorimotor synaptic plasticity and abnormalities in BG neuronal firing have supported the idea of dystonia being a BG disorder. Surprisingly, later studies have highlighted that 
systems other than motor, like somatosensory pathways and cerebellum, are involved in its pathophysiology. This body of evidence suggests that dystonia does not result from dysfunction of a single brain region, but most likely from the dysfunction of a network (Jinnah et al. 2017; Balint et al. 2018). This explains why it is so difficult to identify a single pathological locus in dystonia and is in line with evidence that secondary dystonia can be caused by lesions in $B G$, as well as other structures, in particular the thalamus, brainstem and cerebellum. The network model posits that all subtypes of dystonia result from a network disorder that includes the BG, cerebellum, thalamus, and sensorimotor cortex (Figure 1); however, the precise role and the relevance of each component and the distribution of brain changes have not yet been elucidated. According to the model, dystonia can be produced by a single or multiple nodes dysfunction, in addition to an abnormal interplay among the nodes (Jinnah et al. 2017). The cerebellum seems to have a major role in this regard, as confirmed by animal studies (Quartarone et al. 2019). Indeed, the cerebellum relays proprioceptive information to M1 and contributes to analysis of its spatial and temporal aspects; this processing is deranged in dystonia (Quartarone et al. 2019). Despite this notion being established, electrophysiological data on cerebellar dysfunction in dystonia are still inconclusive, likely because of technical limitations and difficulty of obtaining direct evidence of cerebellar activity in humans.

Considering the network model, it is plausible that the various forms of dystonia (e.g. focal, generalised, and task-specific) reflect derangement at different levels of the network. This might explain the incongruent results among studies investigating patients with different clinical manifestations. Another explanation of this inconsistency may reside in a more complex matter. In recent studies, the "canonical" findings of dystonia, such as reduced inhibition and increased plasticity, have been questioned, giving therefore further importance to the network model. For instance, in some studies SICl has been found to be normal (Kojovic et al. 2013; Ganos et al. 2017; Govert et al. 2020) and others have shown a variable response to plasticity protocols in patients with dystonia (Sadnicka et al. 2014a). The lack of reproducibility of some results can be attributed to the different reliability of the TMS measures, but the interindividual variability to plasticity-inducing paradigm needs also to be taken into account (Latorre et al. 2019). Nevertheless, while to consider some of the most common electrophysiological abnormalities found in dystonia simply as disease "tags" would be overly simplistic, there is no doubt they can help us to draw inferences on the mechanisms causing dystonia (Latorre et al. 2019). In the light of the data and speculations offered here, a unifying theory for dystonia, supported by the network model theory, might be tempted (Conte et al. 2019). Reduced inhibition, as demonstrated by reliable measures such as spinal reciprocal inhibition and blink reflex inhibition, could destabilise circuits' control and therefore cause excess activity, that induces dystonic muscle contraction. This is reinforced by deranged processing of the 
somatosensory input, as supported by reduced somatosensory intracortical inhibition, that may contribute to scarce spatial and temporal discrimination and abnormal sensorimotor integration. In an otherwise normal system, these changes might be compensated; however, the instability of the plasticity control mechanisms might fail in their intent to counteract the excitability imbalance, eventually leading to the expression of dystonia (Figure 1). Although the cerebellum is certainly involved in this network dysfunction, it is still not completely clear what its role in humans is.

In conclusion, with electrophysiology we have been able to establish the pathophysiological basis of this fascinating disorder; not only to fulfil our genuine curiosity on its underlying mechanisms but also with the intent to open very promising treatment scenarios.

\section{Financial Disclosure:}

KPB has received grant support from Horizon 2020 EU grant 634821 and honoraria/financial support to speak/attend meetings from GSK, Boehringer-Ingelheim, Ipsen, Merz, Sun Pharma, Allergan, Teva, Lundbeck and Orion pharmaceutical companies. KPB receives royalties from Oxford University press and a stipend for MDCP editorship. This research study was supported by the National Institute for Health Research University College London Hospitals Biomedical Research Centre and the Edmond J. Safra Philanthropic Foundation All the other authors have no disclosures.

Conflict of Interest: The authors declare that they have no conflict of interest

Funding sources for study: none

Table 1. Electrophysiological characteristics of dystonia

BRC: blink recovery cycle, CBI: cerebellar-brain-inhibition EBCC: eyeblink classic conditioning, GPi/e: internal/external globus pallidus, HF-RSS: high-frequency repetitive somatosensory stimulation, HFO: high-frequency oscillations, IHI: interhemispheric inhibition, RI: reciprocal inhibition, rTMS: repetitive transcranial magnetic stimulation, S1: primary somatosensory cortex, SEP: somatosensory evoked potentials, SICl: short-interval intracortical inhibition, SP: silent period, STDT: somatosensory temporal discrimination threshold, tDCS: transcranial direct current stimulation, TVR: tonic vibration reflex.

Figure 1. Representation of the pathophysiology of dystonia according to the network model The nodes of the network are highlighted with blue ovals and connected by blue arrows (which represent functional, rather than anatomical, connections). A schematic explanation of a unifying theory for dystonia, as discussed in the text, is offered in the boxes.

M1: primary motor cortex, S1: primary somatosensory cortex 


\section{REFERENCES}

Antelmi E, Di Stasio F, Rocchi L, et al. (2016) Impaired eye blink classical conditioning distinguishes dystonic patients with and without tremor. Parkinsonism Relat Disord 31:23-27 doi: 10.1016/j.parkreldis.2016.06.011

Antelmi E, Erro R, Rocchi L, et al. (2017) Neurophysiological correlates of abnormal somatosensory temporal discrimination in dystonia. Mov Disord 32:141-148 doi: 10.1002/mds.26804

Avanzino L, Martino D, van de Warrenburg BP, et al. (2008) Cortical excitability is abnormal in patients with the "fixed dystonia" syndrome. Mov Disord 23:646-652 doi: 10.1002/mds.21801

Balint B, Mencacci NE, Valente EM, et al. (2018) Dystonia. Nat Rev Dis Primers 4:25 doi: 10.1038/s41572-018-0023-6

Bara-Jimenez W, Catalan MJ, Hallett M, Gerloff C (1998) Abnormal somatosensory homunculus in dystonia of the hand. Ann Neurol 44:828-831 doi: 10.1002/ana.410440520

Bara-Jimenez W, Shelton P, Sanger TD, Hallett M (2000) Sensory discrimination capabilities in patients with focal hand dystonia. Ann Neurol 47:377-380

Barow E, Neumann WJ, Brucke C, et al. (2014) Deep brain stimulation suppresses pallidal low frequency activity in patients with phasic dystonic movements. Brain 137:3012-3024 doi: 10.1093/brain/awu258

Beck S, Shamim EA, Richardson SP, Schubert M, Hallett M (2009) Inter-hemispheric inhibition is impaired in mirror dystonia. Eur J Neurosci 29:1634-1640 doi: 10.1111/j.14609568.2009.06710.x

Berardelli A, Rothwell JC, Day BL, Marsden CD (1985) Pathophysiology of blepharospasm and oromandibular dystonia. Brain 108 ( Pt 3):593-608 doi: 10.1093/brain/108.3.593

Brighina F, Romano M, Giglia G, Saia V, Puma A, Giglia F, Fierro B (2009) Effects of cerebellar TMS on motor cortex of patients with focal dystonia: a preliminary report. Exp Brain Res 192:651656 doi: 10.1007/s00221-008-1572-9

Chen CC, Kuhn AA, Trottenberg T, Kupsch A, Schneider GH, Brown P (2006) Neuronal activity in globus pallidus interna can be synchronized to local field potential activity over $3-12 \mathrm{~Hz}$ in patients with dystonia. Exp Neurol 202:480-486 doi: 10.1016/j.expneurol.2006.07.011

Chen R, Cros D, Curra A, et al. (2008) The clinical diagnostic utility of transcranial magnetic stimulation: report of an IFCN committee. Clin Neurophysiol 119:504-532 doi: 10.1016/j.clinph.2007.10.014

Chen R, Wassermann EM, Canos M, Hallett M (1997) Impaired inhibition in writer's cramp during voluntary muscle activation. Neurology 49:1054-1059

Conte A, Rocchi L, Latorre A, Belvisi D, Rothwell JC, Berardelli A (2019) Ten-Year Reflections on the Neurophysiological Abnormalities of Focal Dystonias in Humans. Mov Disord 34:1616-1628 doi: $10.1002 / \mathrm{mds} .27859$

DeLong MR (1990) Primate models of movement disorders of basal ganglia origin. Trends Neurosci 13:281-285

Edwards MJ, Huang YZ, Mir P, Rothwell JC, Bhatia KP (2006) Abnormalities in motor cortical plasticity differentiate manifesting and nonmanifesting DYT1 carriers. Mov Disord 21:2181-2186 doi: 10.1002/mds. 21160

Edwards MJ, Huang YZ, Wood NW, Rothwell JC, Bhatia KP (2003) Different patterns of electrophysiological deficits in manifesting and non-manifesting carriers of the DYT1 gene mutation. Brain 126:2074-2080 doi: 10.1093/brain/awg209

Elbert T, Candia V, Altenmuller E, et al. (1998) Alteration of digital representations in somatosensory cortex in focal hand dystonia. Neuroreport 9:3571-3575 doi: 10.1097/00001756-19981116000006

Erro R, Rocchi L, Antelmi E, et al. (2018) High frequency somatosensory stimulation in dystonia: Evidence fordefective inhibitory plasticity. Mov Disord doi: 10.1002/mds.27470 
Erro R, Rocchi L, Antelmi E, Palladino R, Tinazzi M, Rothwell J, Bhatia KP (2016) High frequency repetitive sensory stimulation improves temporal discrimination in healthy subjects. Clin Neurophysiol 127:817-820 doi: 10.1016/j.clinph.2015.06.023

Espay AJ, Morgante F, Purzner J, Gunraj CA, Lang AE, Chen R (2006) Cortical and spinal abnormalities in psychogenic dystonia. Ann Neurol 59:825-834 doi: 10.1002/ana.20837

Fiorio M, Tinazzi M, Bertolasi L, Aglioti SM (2003) Temporal processing of visuotactile and tactile stimuli in writer's cramp. Ann Neurol 53:630-635 doi: 10.1002/ana.10525

Ganos C, Ferre ER, Marotta A, Kassavetis P, Rothwell J, Bhatia KP, Haggard P (2017) Cortical inhibitory function in cervical dystonia. Clin Neurophysiol doi: 10.1016/j.clinph.2017.11.020

Garraux G, Bauer A, Hanakawa T, Wu T, Kansaku K, Hallett M (2004) Changes in brain anatomy in focal hand dystonia. Ann Neurol 55:736-739 doi: 10.1002/ana.20113

Govert F, Becktepe J, Balint B, et al. (2020) Temporal discrimination is altered in patients with isolated asymmetric and jerky upper limb tremor. Mov Disord 35:306-315 doi: $10.1002 /$ mds. 27880

Huang YZ, Edwards MJ, Bhatia KP, Rothwell JC (2004) One-Hz repetitive transcranial magnetic stimulation of the premotor cortex alters reciprocal inhibition in DYT1 dystonia. Mov Disord 19:54-59 doi: 10.1002/mds.10627

Hutchison WD, Lang AE, Dostrovsky JO, Lozano AM (2003) Pallidal neuronal activity: implications for models of dystonia. Ann Neurol 53:480-488 doi: 10.1002/ana.10474

Jinnah HA, Neychev V, Hess EJ (2017) The Anatomical Basis for Dystonia: The Motor Network Model. Tremor Other Hyperkinet Mov (N Y) 7:506 doi: 10.7916/D8V69X3S

Kaji R, Rothwell JC, Katayama M, et al. (1995) Tonic vibration reflex and muscle afferent block in writer's cramp. Ann Neurol 38:155-162 doi: 10.1002/ana.410380206

Kojovic M, Parees I, Kassavetis P, et al. (2013) Secondary and primary dystonia: pathophysiological differences. Brain 136:2038-2049 doi: 10.1093/brain/awt150

Latorre A, Rocchi L, Berardelli A, Bhatia KP, Rothwell JC (2019) The interindividual variability of transcranial magnetic stimulation effects: Implications for diagnostic use in movement disorders. Mov Disord 34:936-949 doi: 10.1002/mds.27736

Marsden CD, Rothwell JC (1987) The physiology of idiopathic dystonia. Can J Neurol Sci 14:521-527 doi: $10.1017 / \mathrm{s} 031716710003804 x$

Meunier S, Garnero L, Ducorps A, et al. (2001) Human brain mapping in dystonia reveals both endophenotypic traits and adaptive reorganization. Ann Neurol 50:521-527 doi: 10.1002/ana.1234

Meunier S, Russmann H, Shamim E, Lamy JC, Hallett M (2012) Plasticity of cortical inhibition in dystonia is impaired after motor learning and paired-associative stimulation. Eur J Neurosci 35:975-986 doi: 10.1111/j.1460-9568.2012.08034.x

Mitchell IJ, Luquin R, Boyce S, Clarke CE, Robertson RG, Sambrook MA, Crossman AR (1990) Neural mechanisms of dystonia: evidence from a 2-deoxyglucose uptake study in a primate model of dopamine agonist-induced dystonia. Mov Disord 5:49-54 doi: 10.1002/mds.870050113

Monaco J, Rocchi L, Ginatempo F, D'Angelo E, Rothwell JC (2018) Cerebellar Theta-Burst Stimulation Impairs Memory Consolidation in Eyeblink Classical Conditioning. Neural Plast 2018:6856475 doi: $10.1155 / 2018 / 6856475$

Murase N, Kaji R, Shimazu H, et al. (2000) Abnormal premovement gating of somatosensory input in writer's cramp. Brain 123 ( Pt 9):1813-1829 doi: 10.1093/brain/123.9.1813

Nakashima K, Rothwell JC, Day BL, Thompson PD, Shannon K, Marsden CD (1989) Reciprocal inhibition between forearm muscles in patients with writer's cramp and other occupational cramps, symptomatic hemidystonia and hemiparesis due to stroke. Brain 112 ( Pt 3):681-697 doi: $10.1093 /$ brain/112.3.681

Nakashima K, Rothwell JC, Thompson PD, et al. (1990) The blink reflex in patients with idiopathic torsion dystonia. Arch Neurol 47:413-416 doi: 10.1001/archneur.1990.00530040055019 
Nelson AJ, Hoque T, Gunraj C, Ni Z, Chen R (2010) Impaired interhemispheric inhibition in writer's cramp. Neurology 75:441-447 doi: 10.1212/WNL.0b013e3181ebdda0

Neumann WJ, Jha A, Bock A, et al. (2015) Cortico-pallidal oscillatory connectivity in patients with dystonia. Brain 138:1894-1906 doi: 10.1093/brain/awv109

Newby RE, Thorpe DE, Kempster PA, Alty JE (2017) A History of Dystonia: Ancient to Modern. Mov Disord Clin Pract 4:478-485 doi: 10.1002/mdc3.12493

Quartarone A, Bagnato S, Rizzo V, et al. (2003) Abnormal associative plasticity of the human motor cortex in writer's cramp. Brain 126:2586-2596 doi: 10.1093/brain/awg273

Quartarone A, Cacciola A, Milardi D, et al. (2019) New insights into cortico-basal-cerebellar connectome: clinical and physiological considerations. Brain doi: 10.1093/brain/awz310

Quartarone A, Rizzo V, Bagnato S, et al. (2005) Homeostatic-like plasticity of the primary motor hand area is impaired in focal hand dystonia. Brain 128:1943-1950 doi: 10.1093/brain/awh527

Quartarone A, Rizzo V, Terranova C, et al. (2009) Abnormal sensorimotor plasticity in organic but not in psychogenic dystonia. Brain 132:2871-2877 doi: 10.1093/brain/awp213

Quartarone A, Siebner HR, Rothwell JC (2006) Task-specific hand dystonia: can too much plasticity be bad for you? Trends Neurosci 29:192-199 doi: 10.1016/j.tins.2006.02.007

Ridding MC, Inzelberg R, Rothwell JC (1995a) Changes in excitability of motor cortical circuitry in patients with Parkinson's disease. Ann Neurol 37:181-188 doi: 10.1002/ana.410370208

Ridding MC, Sheean G, Rothwell JC, Inzelberg R, Kujirai T (1995b) Changes in the balance between motor cortical excitation and inhibition in focal, task specific dystonia. J Neurol Neurosurg Psychiatry 59:493-498

Rocchi L, Erro R, Antelmi E, et al. (2017) High frequency somatosensory stimulation increases sensori-motor inhibition and leads to perceptual improvement in healthy subjects. Clin Neurophysiol 128:1015-1025 doi: 10.1016/j.clinph.2017.03.046

Rothwell JC, Day BL, Obeso JA, Berardelli A, Marsden CD (1988) Reciprocal inhibition between muscles of the human forearm in normal subjects and in patients with idiopathic torsion dystonia. Adv Neurol 50:133-140

Rothwell JC, Huang YZ (2003) Systems-level studies of movement disorders in dystonia and Parkinson's disease. Curr Opin Neurobiol 13:691-695 doi: 10.1016/j.conb.2003.10.006

Rothwell JC, Obeso JA, Day BL, Marsden CD (1983) Pathophysiology of dystonias. Adv Neurol 39:851863

Ruge D, Cif L, Limousin P, et al. (2011a) Shaping reversibility? Long-term deep brain stimulation in dystonia: the relationship between effects on electrophysiology and clinical symptoms. Brain 134:2106-2115 doi: 10.1093/brain/awr122

Ruge D, Tisch S, Hariz MI, et al. (2011b) Deep brain stimulation effects in dystonia: time course of electrophysiological changes in early treatment. Mov Disord 26:1913-1921 doi: $10.1002 / \mathrm{mds} .23731$

Sadnicka A, Hamada M, Bhatia KP, Rothwell JC, Edwards MJ (2014a) A reflection on plasticity research in writing dystonia. Mov Disord 29:980-987 doi: 10.1002/mds.25908

Sadnicka A, Kimmich O, Pisarek C, et al. (2013) Pallidal stimulation for cervical dystonia does not correct abnormal temporal discrimination. Mov Disord 28:1874-1877 doi: 10.1002/mds.25581

Sadnicka A, Patani B, Saifee TA, et al. (2014b) Normal motor adaptation in cervical dystonia: a fundamental cerebellar computation is intact. Cerebellum 13:558-567 doi: 10.1007/s12311014-0569-0

Sadnicka A, Teo JT, Kojovic M, et al. (2015) All in the blink of an eye: new insight into cerebellar and brainstem function in DYT1 and DYT6 dystonia. Eur J Neurol 22:762-767 doi: 10.1111/ene.12521

Sattler V, Dickler M, Michaud M, Meunier S, Simonetta-Moreau M (2014) Does abnormal interhemispheric inhibition play a role in mirror dystonia? Mov Disord 29:787-796 doi: $10.1002 /$ mds. 25768 
Schwingenschuh P, Katschnig P, Edwards MJ, Teo JT, Korlipara LV, Rothwell JC, Bhatia KP (2011) The blink reflex recovery cycle differs between essential and presumed psychogenic blepharospasm. Neurology 76:610-614 doi: 10.1212/WNL.0b013e31820c3074

Sheehy MP, Rothwell JC, Marsden CD (1988) Writer's cramp. Adv Neurol 50:457-472

Silberstein P, Kuhn AA, Kupsch A, et al. (2003) Patterning of globus pallidus local field potentials differs between Parkinson's disease and dystonia. Brain 126:2597-2608 doi: 10.1093/brain/awg267

Starr PA, Rau GM, Davis V, et al. (2005) Spontaneous pallidal neuronal activity in human dystonia: comparison with Parkinson's disease and normal macaque. J Neurophysiol 93:3165-3176 doi: 10.1152/jn.00971.2004

Teo JT, van de Warrenburg BP, Schneider SA, Rothwell JC, Bhatia KP (2009) Neurophysiological evidence for cerebellar dysfunction in primary focal dystonia. J Neurol Neurosurg Psychiatry 80:80-83 doi: 10.1136/jnnp.2008.144626

Tinazzi M, Fiorio M, Stanzani C, et al. (2006) Temporal discrimination of two passive movements in writer's cramp. Mov Disord 21:1131-1135 doi: 10.1002/mds.20892

Vitek JL (2002) Pathophysiology of dystonia: a neuronal model. Mov Disord 17 Suppl 3:S49-62 doi: 10.1002/mds.10142

Vitek JL, Chockkan V, Zhang JY, et al. (1999) Neuronal activity in the basal ganglia in patients with generalized dystonia and hemiballismus. Ann Neurol 46:22-35 doi: 10.1002/15318249(199907)46:1<22::aid-ana6>3.0.co;2-z

Vitek JL, Delong MR, Starr PA, Hariz MI, Metman LV (2011) Intraoperative neurophysiology in DBS for dystonia. Mov Disord 26 Suppl 1:S31-36 doi: 10.1002/mds.23619

Wichmann T, Dostrovsky JO (2011) Pathological basal ganglia activity in movement disorders. Neuroscience 198:232-244 doi: 10.1016/j.neuroscience.2011.06.048 